under-represented in clinical services. An open-access service in the North of England provides a breadth of support to people affected by palliative diagnoses, without requiring clinical referral, with dedicated support to address some of these inequalities. We report on an evaluation of this service.

Method This study was conducted as a secondary data analysis of an annual service evaluation. Characteristic data of service users was collected through an anonymous cross-sectional survey. One-hundred and fifteen responses were acquired via convenience sampling over the period of one week. Data was compared against the characteristics of local specialist palliative care services, local census data and historical MDS data through descriptive and inferential techniques (chi-squared test).

Findings The open-access service was found to have a significantly higher proportion of non-cancer patients $(75 \%)$ compared with clinical services (27\%) and MDS data (24\%), highlighting that this is a potentially valuable approach to improving access for this under-represented group (p0.05). Conversely, attendees were more likely to be female $(<0.001)$, under $75(<0.001)$, live in the local area $(<0.001)$ and have religious beliefs $(<0.01)$, highlighting areas for further development.

Conclusion This service evaluation has highlighted a potential approach for improving access to specialist support for people with non-malignant disease and members of minority ethnic groups; formal research evaluation is recommended. Outstanding challenges include broadening access for men, older people, non-religious people and the wider area.

\section{INTRODUCING A LEARNING FROM DEATHS MEETING IN A HOSPICE}

Molly Bird, Esther Reilly, Lisa Kennedy, Helen Smith. Birmingham St Mary's Hospice

\subsection{6/spcare-2020-PCC.133}

Introduction 'Learning from deaths' is a monthly meeting open to all clinical staff from across hospice services. It is a forum to discuss, reflect on and learn from the deaths of our patients.

Method The meeting was established as there was no formal forum to discuss deaths in the hospice and a positive experience attending a similar event gave the forward momentum and inspiration to create this meeting. Suggestions of deaths to be discussed can be made by any clinician and may include deaths which have been challenging or traumatic as well as deaths which have been examples of good practice. An overview of the case is presented including what happened, what went well and what could be improved. Senior clinicians are present to guide the discussion, ensuring a positive focus is maintained and assisting with identifying the learning for the future.

Results This meeting was launched in July 2019. There has been an encouraging turnout with an average of 22 attendees and positive feedback has been given. Particular comment has been made regarding the tone of the meeting and it being a safe, reflective non-judgmental space. Another strength is noted as learning being focused on improving future practice and care. A learning $\log$ is gathered during the meetings to ensure that discussion is put into action. For example following one discussion a series of educational sessions were organised about the withdrawal of non-invasive ventilation.

Conclusion Learning from deaths is now considered a vital part of clinical practice, support for staff and an opportunity for learning. In the future invitations to attend the meeting could be extended to clinicians from outside the hospice who were involved in the individual's care e.g. General Practitioners, District Nurses.

\section{APPROACHING THE END OF LIFE: ARE REFERRALS TO HOSPITAL SPECIALIST PALLIATIVE CARE TEAM ARRIVING TOO LATE?}

Elaine G Boland, Kate Hardcastle, Margaret Simkiss, Liz Lawson, Fliss EM Murtagh. Hull University Teaching Hospitals NHS Trust, Wolfson Palliative Care Research Centre, Hull York Medical School

\subsection{6/spcare-2020-PCC.134}

Introduction Late referrals to specialist palliative care teams (SPCT) limit the ability of and time for SPCTs to improve symptoms and reduce distress for patients and their families. We aimed to identify all patients referred to the hospital SPCT who died before being seen during 2018 to:

1. Assess if uncontrolled symptoms were present

2. Ensure SPCT responded rapidly

3. Identify ways to support wards with timely referrals and reduce late referrals.

Methods Design: Audit with case-notes review

Part 1: we identified all hospital patients referred to SPCT who died before being seen, and assessed the hospitals/wards/ patient characteristics to identify patterns. We reviewed referral time and our response times/telephone advice.

Part 2: Detailed case-notes review of 15\% (randomly selected) patients who died before being seen by the SPCT to assess recognition of dying, see if these dying patients had specialist palliative care needs and if symptoms/distress were controlled or not.

Results Part 1: In 2018, the SPCT reviewed 1520 patients across the acute trust. 87 (5.6\%) patients died before being seen by the SPCT. Of those, 19 patients (21\%) were referred and died outside SPCT working hours. Referrals came from 25 different wards. 42 (37\%) patients died within 6 hours of referral. Telephone advice was given to healthcare professionals for 37 patients (42\%). Most patients were over 65 years old; the common causes of death were pneumonia and cancer.

Part 2: 15 case-notes had a detailed review. Median length of stay was 9 days (range 2-40days). Most patients were documented as dying only in the last 2 days. 5 patients had no SPC needs whilst 10 patients had uncontrolled symptoms. Conclusion Promoting earlier recognition of dying and a more integrated approach of palliative care alongside active interventions could optimise symptom management and reduce distress towards end of life for patients dying in hospitals.

\section{COMPASSIONATE CARE BED IN PARTNERSHIP WITH INTERMEDIATE CARE SERVICES - NORTH MANCHESTER}

Lindy Brooks, Elizabeth A O'Brien, Alicia Waite. Manchester Macmillan Supportive and Palliative Care Service - North Locality

\subsection{6/spcare-2020-PCC.135}

Background The North Manchester Palliative Care Support Service (MMSPCSNL) was introduced in April 2016 with a significant remodelling of the previous provision. The service 
expanded local capacity and transformed the quality and experience of supportive and palliative care Some patients were identified as being not in need of specialist hospice beds or acute hospital admission but had non-complex requirements that could not be met in their ordinary home environment. A single bed within an Intermediate Care Unit was made available to support this care.

Methods Patients with non-complex needs are considered for admission to this bed if they are known to the MMSPCSNL and assessed by a band 7 clinician or doctor within the team at time of need. The bed is not suitable for individuals with complex needs or requiring daily medical review/input. During their admission they are supported by the staff within the unit as for any other inpatient in addition to regular review by the specialist palliative care team.

Results Since the pilot commenced, the skills and experience of the staff in the unit has increased with admission of patients with varying needs. The utilisation of the bed has been for multiple reasons from rehabilitation following cancer treatment in acute hospitals, to social crisis admissions, noncomplex end of life care and for combined reasons. During the first 12 months of the pilot 8 patients were supported with an admission length ranging from 12 to 65 days. 6 were discharged, 2 died in the compassionate bed as their preferred place of care.

Conclusion This pilot has achieved multiple positive outcomes such as appropriate care for patients in social crisis preventing acute hospital admissions, enhanced skills for unit staff and flexibility of approach with all teams involved. Funding is ongoing - the pilot has received favourable results and comments.

\section{CONSIDERING LIFE IMPACT OUTCOMES ON THE EARLY IDENTIFICATION OF PATIENTS IN NEED OF PALLIATIVE CARE ON THE DEVELOPMENT OF PREDICTIVE MODELS}

Mateos MJ Caballero, MS Giménez Campos, V Blanes-Selva, A Fernandes, JM GarcíaGiménez, A Duarte-Martinez, ME Gas López. Joint Research Unit in ICT applied to reengineering socio-sanitary process.Departament de Salut Valencia La Fe; Departament de Salut Valencia La Fe; Biomedical Data Science Lab ITACA Institute. Universitat Politecnica de Valencia; Santa Casa da Misericordi

\subsection{6/spcare-2020-PCC.136}

Introduction Stratification and predictive models have been proposed to support clinical decisions or to identify vulnerability-based sub-populations of complex patients. In particular, predicting mortality also has been one of the initiatives proposed on the prediction of palliative care (PC) needs. However, models considering life impact outcomes, such as negative effects due to illnesses, may be more able to contribute to identifying older patients who may benefit from early PC. The aim of this study was to review the current state of the art on health indicators which could suggest a declining trajectory of end-of-life in patients aged $>65$ with chronic disease(s).

Method A literature review was carried out in PubMed-Medline (February 20, 2019). Two research questions (RQ) were formulated (RQ1: mortality or survival prediction; RQ2: identification of patients with PC needs). Study selection was performed independently by two reviewers under the following inclusion criteria: subject sample aged $>65$, with chronic disease profile, published in English. Outcomes were categorised using the Core Outcome Measures in Effectiveness TrialsCOMET taxonomy, specifically the core area: Life Impact.

Results The keyword search gathered 712 papers for RQ1 and 696 for RQ2. After screening papers and eliminating duplicates, 101 studies were considered for retrieving information. We identified 1723 outcomes candidates but, after decoding abbreviations and concealing terms, only 484 remained. Of these, 83 terms were aligned with the core area Life Impact, being grouped under the following subdomains: cognitive $(n=13)$, physical $(n=28)$, social $(n=16)$ and emotional $(n=10)$ functioning; quality of life $(n=1)$; perceived health status $(n=6)$ and care delivered $(n=9)$.

Conclusion The literature review allowed us to collect updated life impact outcomes related to repercussions of illnesses which may guide the development of predictive modelling beyond a mortality approaching paradigm under the clinical setting mentioned.

\section{PATIENTS WITH PALLIATIVE CARE NEEDS NOT SO DISSIMILAR TO THOSE OF FRAILTY: TIME FOR SHARING APPROACHES}

Declan A Cawley, Jasmine Mann, Beverley Pryke. St Michael's Hospice, Medway NHS Foundation Trust, Wisdom Hospice

\subsection{6/spcare-2020-PCC.137}

Background Frailty within recent years has become a priority for healthcare providers looking at how anticipatory care plans can influence the potential and avoidable possibility of hospital admissions. Similarly, supportive and palliative care aims to meet individuals' preferences and wishes with hospital admission avoidance a high priority. The hospital setting is therefore an ideal opportunity when needs are identified to set in motion anticipatory/advance care plans, specifically looking at admission avoidance.

Aim To explore the needs of a hospital based supportive and palliative care caseload in conjunction with those of frailty.

Methods A retrospective case-note review of individuals referred to a hospital based supportive and palliative care team (HSPC) and frailty team (F) using standardised data collection sheet.

Results 200 case-notes were reviewed, 100 from each team. Individuals had broadly similar needs with the Clinical Frailty Scale (CFS) scoring 5 or above, 92\% from HSPC and $87 \%$ from frailty. The age differed unsurprisingly (74yrs vs. $84 \mathrm{yrs}$ (average)) with similar numbers of individuals having had polypharmacy (> 5 or more)(69\% vs. $71 \%)$. Significant differences were noted in the predominating diagnosis (cancer $-69 \%$ vs. $3 \%)$, multi-morbidity ( $41 \%$ vs. $67 \%$ ) and those residing in care homes (2\% vs. 19\%).

Conclusions The findings highlight broadly similar needs for individuals whether they are under a supportive and palliative care team or that of a frailty team within a hospital setting. In particular multi-morbidity, polypharmacy and needs as assessed by Clinical Frailty Score (CFS) were broad similar. The opportunity lies within healthcare providers using similar processes and pooling resources to meet the needs of frailty as well as those of supportive and palliative care. The use of a universal holistic assessment tool is a priority for further exploration within these teams and the individuals they care for. 